\title{
Efectividad de aprendizaje por competencias en ingeniería del software
}

\section{Learning effectiveness by software engineering competencies}

\author{
Wilian Richart Delgado Muentes \\ wiliandelgado@gmail.com \\ Universidad Laica Eloy Alfaro de Manabí, Manta \\ Ecuador \\ https://orcid.org/0000-0002-5136-0677
}

Recibido: 1 de agosto de 2019

Aprobado: 1 de septiembre de 2019

\begin{abstract}
RESUMEN
La investigación tuvo por objetivo determinar la efectividad de aprendizaje por competencias en ingeniería del software. La población estuvo conformada por 21 estudiantes para el grupo control y 18 para el grupo experimental de la universidad Laica Eloy Alfaro de Manabí (ULEAM). Se evidencia que el grupo experimental en pos prueba obtuvo 0,83 de significancia bilateral, lo cual implica que existió cambio en relación a la pre prueba, y al grupo control, por lo tanto se acepta la hipótesis verdadera. Los estudiantes objeto de estudio han desarrollado competencias genéricas como liderazgo gerencial - social en detectar y asumir el reto de solucionar una problemática real, mediante el diseño de un software para la industria atunera de Manta.
\end{abstract}

Descriptores: Educación alternativa; Experimento educacional; Investigación pedagógica, Modelo educacional.

\begin{abstract}
The research aimed to determine the effectiveness of learning by competencies in software engineering. The population consisted of 21 students for the control group and 18 for the experimental group of the Laica Eloy Alfaro University in Manabí (ULEAM). It is evident that the experimental group in post-test obtained 0.83 of bilateral significance, which implies that there was a change in relation to the pre-test, and the control group, therefore the true hypothesis is accepted. The students under study have developed generic skills such as managerial - social leadership in detecting and taking on the
\end{abstract}




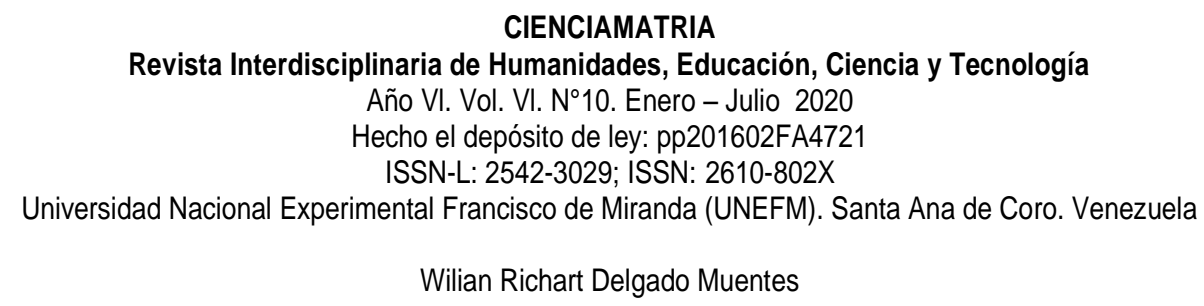

challenge of solving a real problem, by designing software for the Manta tuna industry.

Descriptors: Alternative education; Educational experiments; Educational research; Educational models.

\section{INTRODUCCIÓN}

El aprendizaje por competencias busca la formación integral de los estudiantes, mediante la adopción de perspectiva integradora de acciones que conlleven a la resolución de problemas concretos en sostenimiento de una conducta ética, de allí que el Ministerio de Educación de El Salvador (2008), señale que este aprendizaje procura:

1. El saber referido a conceptos, hechos, datos, principios, definiciones, esquemas, secuencias instruccionales, entre otros. Es equivalente a los contenidos declarativos o conceptuales.

2. El saber hacer es decir, las habilidades, y destrezas que el individuo utiliza en una actuación determinada con base en los conocimientos internalizados. Es equivalente a los contenidos procedimentales.

3. El saber ser y convivir o sea, el comportamiento o conducta observable de un individuo al resolver una tarea. Refleja los valores y las actitudes que se ponen en juego al llevar a cabo la actividad. Es equivalente a los contenidos actitudinales.

En cada área del saber se establecen competencias y sub competencias con la finalidad de generar un proceso integrador de acciones que permitan realizar en el estudiante la consecución de operaciones concatenadas entre sí como efecto integrador del conocimiento, para lo cual se necesita fusionar teoría - práctica con el saber producto de la experiencia, así se genera un estudiante pertinente en relación a los postulados de la educación 2030 y a los principios constitucionales, políticas educativas del Ecuador. En este sentido, Frandeschin (2018), plantea que este enfoque se caracteriza por:

1. La capacidad del estudiante de aprovechar sus conocimientos previos. Al evaluarse su nivel de comprensión de una temática y su capacidad de aplicarla en ejercicios teóricos y prácticos, el Aprendizaje basado en competencias no requiere 


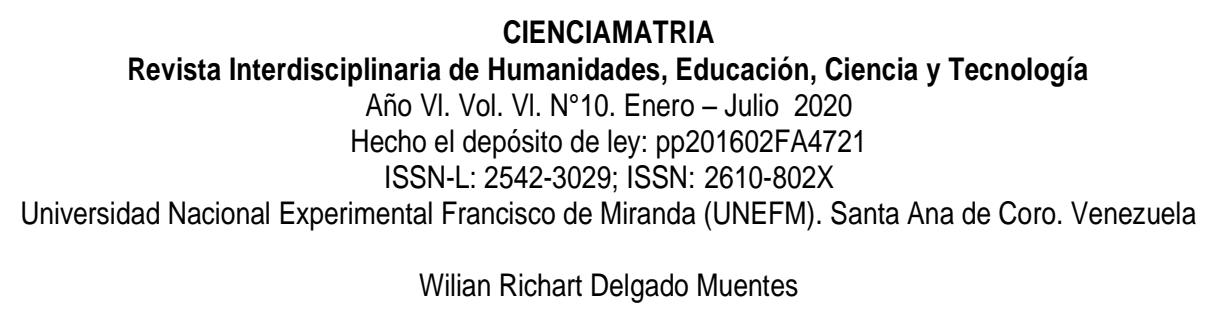

que los estudiantes se apeguen a un programa en particular para poder avanzar. Así, se optimiza su tiempo, ya no deben repetir el aprendizaje de conocimientos previos, ni tener que tramitar tediosas equivalencias para poder avanzar en sus carreras.

2. Flexibilidad y autogestión. Una de las grandes ventajas de este modelo es que exime a los estudiantes de tener que seguir programas rígidos, y adaptarse a tiempos de cursada como trimestres o semestres, y les permite seguir su propio ritmo, superando las etapas según su capacidad de asimilar el conocimiento y aprender a ponerlo en práctica.

3. Transparencia y consistencia en las habilidades de los graduados. Al requerir un mayor dominio de las temáticas por parte de los egresados, esta metodología puede ofrecer mejores garantías de su capacidad de adaptarse a determinadas posiciones y hacer ciertos trabajos. Si bien los títulos regulares hoy pueden ofrecer una cierta orientación en cuanto a los conocimientos de los profesionales, éstos no ofrecen garantías acerca de su dominio de cada uno de los tópicos estudiados durante sus carreras.

4. Formación integral y desarrollo de ciudadanía. Otra ventaja de esta metodología es que requiere que, para aprobar, los estudiantes desarrollen una gran cantidad de habilidades pensamiento lógico, aprendizaje continuo, y comunicación, algo que no siempre ocurre en las carreras tradicionales.

Se concibe el aprendizaje por competencias como una alternativa para generar un aprendizaje significativo en la ingeniería del software que permita a los estudiantes, generar soluciones viables en la comunidad cercana a su contexto de estudio, en este sentido, la actual investigación se propone determinar la efectividad de aprendizaje por competencias en ingeniería del software de la universidad Laica Eloy Alfaro de Manabí (ULEAM). 


\section{DESARROLLO}

\section{Aprendizaje por competencias}

El aprendizaje por competencias de acuerdo a Tobón (2009), se clasifica en: 1. competencias básicas, 2. competencias genéricas, 3. Competencias específicas, para lo cual se tiene:

Competencias básicas: Son las competencias fundamentales para vivir en sociedad y desenvolverse en cualquier ámbito laboral. Estas competencias se caracterizan por: (1) constituyen la base sobre la cual se forman los demás tipos de competencias; (2) se forman en la educación básica y media; (3) posibilitan analizar, comprender y resolver problemas de la vida cotidiana; (4) constituyen un eje central en el procesamiento de la información de cualquier tipo (p. 87).

En complemento, Tobón (2009), indica que las competencias básicas se subdividen en:

1. Competencia comunicativa: Comunicar los mensajes acorde con los requerimientos de una determinada situación. Algunas de las actividades que la persona puede realizar en esta competencia es; Interpretar textos atendiendo a las intenciones comunicativas, a sus estructuras y a sus relaciones. Producir textos con sentido, coherencia y cohesión requeridos.

2. Competencia matemática: Resolver problemas con base en el lenguaje y procedimientos de la matemática. Algunas de las actividades que la persona puede realizar en esta competencia se encuentran; Resolver los problemas con base en la formulación matemática requerida por éstos. Así como Interpretar la información que aparece en lenguaje matemático, acorde con los planteamientos conceptuales y metodológicos de esta área.

3. Competencia de autogestión del proyecto ético de vida: consiste en Auto gestionar el proyecto ético de vida acorde con las necesidades vitales personales, las propias competencias y las oportunidades y limitaciones del contexto. Algunas de las actividades que la persona puede realizar en esta competencia se encuentran; Identificar las necesidades vitales personales, las competencias y el contexto. Planificar el proyecto ético de vida identificando las metas a corto, mediano y largo 
plazo, las estrategias para alcanzarlas y los factores de incertidumbre. Autoevaluar de manera constante la forma como se están satisfaciendo las necesidades vitales personales y modificar las estrategias de acción cuando se estime oportuno.

4. Manejo de las Nuevas Tecnologías de la Información y la Comunicación: Manejar las Nuevas Tecnologías de la Información y la Comunicación con base en los requerimientos del contexto. Algunas de las actividades que la persona puede realizar en esta competencia se encuentran; Manejar el computador a nivel de usuario, procesando información en programas básicos (hojas de cálculo, procesador de textos, diseño de presentaciones, etc.). - Comunicarse mediante el uso de internet (correo electrónico, chato video chat, páginas web, etc.); Comunicarse mediante el empleo de la telefonía fija y móvil.

5. Afrontamiento del cambio: Manejar los procesos de cambio en los diferentes escenarios de la vida, acorde con estrategias del plan de vida o de una determinada organización. Algunas de las actividades que la persona puede realizar en esta competencia se encuentran; Implementar estrategias flexibles que permitan manejar los procesos de cambio inesperados. - Modificar planes y proyectos con el fin de manejar los procesos de cambio.

6. Liderazgo: Liderar actividades y proyectos en beneficio personal y de las demás personas, con base en las posibilidades del contexto. Algunas de las actividades que la persona puede realizar en esta competencia se encuentran; Gestionar la consecución de recursos económicos, físicos, materiales y de infraestructura. Motivar a las personas a alcanzar metas mediante el trabajo cooperativo. Gestionar alianzas estratégicas para la realización de actividades.

Las competencias básicas son relacionadas a los aspectos elementales que debe poseer tanto el docente como el estudiante, de ese modo, se diseña el aprendizaje con la finalidad de que estos puedan ser desarrollados transversalmente a lo largo del ciclo 


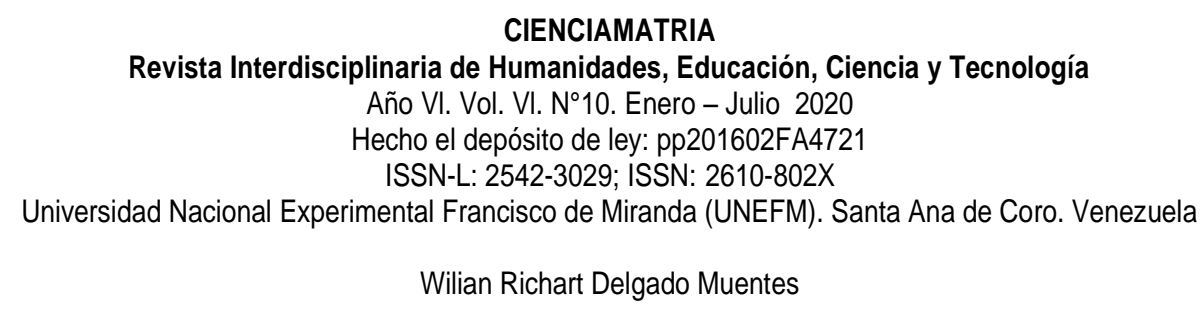

formativo. Así mismo en relación a las competencias genéricas, Corominas (2001) citado por Tobón (2009), plantea que estas competencias:

Son aquellas competencias comunes a varias ocupaciones o profesiones. Por ejemplo, los profesionales de reas tales como la administración de empresas, la contaduría y la economía comparten un conjunto de competencias genéricas tales como: análisis financiero y gestión empresarial. Este tema comienza a ser de gran importancia en la educación universitaria, la cual debe formar en los estudiantes competencias genéricas que les permitan afrontar los continuos cambios del quehacer profesional (p. 91).

En el caso de la ingeniería de software, se comprenden que son las competencias focalizas al manejo de ciertas operaciones organizacionales, entre las cuales, Tobón (2009), plantea lo siguiente:

1. Emprendimiento: Iniciar nuevos proyectos productivos o de mejoramiento de las condiciones de trabajo, con base en los requerimientos organizacionales y las demandas externas. Algunas de las actividades que la persona puede realizar en esta competencia se encuentran; Diseñar nuevos proyectos acorde con los requerimientos de la organización y del contexto empresarial. - Ejecutar los proyectos con referencia a las metas propuestas, el proceso administrativo definido y las condiciones el entorno.

2. Gestión de recursos: Gestionar recursos de diverso tipo con base en los requerimientos de la producción. Algunas de las actividades que la persona puede realizar en esta competencia se encuentran; determinar los recursos que requieren las actividades con base en los requerimientos formulados. - Asignar los recursos económicos y materiales a los procesos y subprocesos, acorde con los requerimientos.

3. Trabajo en equipo: Planificar el trabajo en equipo teniendo como referencia los objetivos estratégicos de la organización. Algunas de las actividades que la persona puede realizar en esta competencia se encuentran; Implementar equipos de trabajo acorde con los requerimientos organizacionales específicos. - Negociar 
conflictos de manera pacífica, teniendo como referencia los requerimientos situacionales.

4. Resolución de problemas: Resolver los problemas planteados por una determinada situación, con base en el logro de los objetivos estratégicos de la organización. Algunas de las actividades que la persona puede realizar en esta competencia se encuentran; Detectar los problemas dentro del marco laboral, teniendo como base el contexto en el cual se presentan y los diferentes actores involucrados. - Implementar acciones concretas para resolver los problemas con base en el contexto laboral y el logro de los objetivos organizacionales.

5.

Estas sub competencias permiten que el estudiante se forme para el ejercicio profesional desde un sentido amplio de gestión organizacional, por otro lado en relación a las competencias específicas Tobón (2009), las define como "aquellas competencias propias de una determinada ocupación o profesión. Tienen un alto grado de especialización, así,, como procesos educativos específicos, generalmente llevados a cabo en programas técnicos, de formación para el trabajo y en educación superior" ( $p$. 93), destacándose las siguientes sub competencias:

1. Diseño del Proyecto Educativo Institucional: Formular el proyecto educativo con base en la filosofía institucional, la política educativa estatal, la normatividad vigente y las necesidades del entorno. Algunas de las actividades que la persona puede realizar en esta competencia se encuentran; Definir el componente teleológico del proyecto educativo con base en los resultados del diagnóstico. - Trazar la estrategia institucional en coherencia con las prioridades establecidas en el proyecto educativo. Orientar la formulación de parámetros e indicadores para la evaluación del proyecto educativo, con base en la filosofía institucional.

2. Liderazgo del Proyecto Educativo Institucional: Liderar el proyecto educativo con base en los indicadores de gestión establecidos, las competencias asignadas y la 


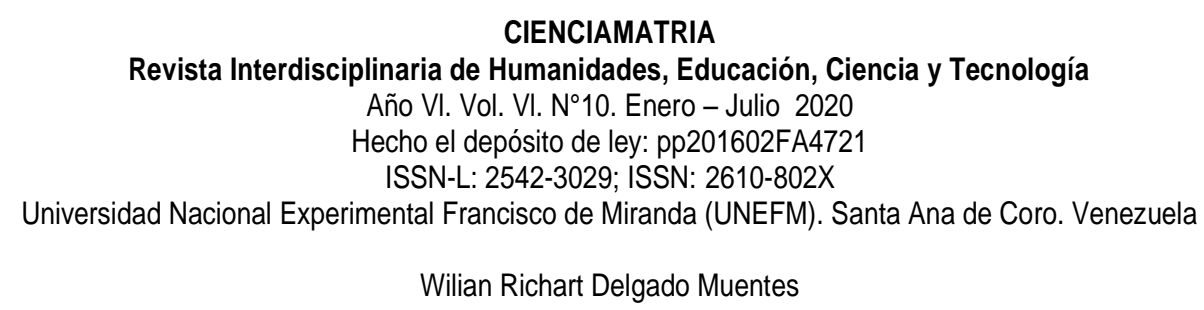

normatividad vigente. Algunas de las actividades que la persona puede realizar en esta competencia se encuentran; Dirigir los procesos educativos con base en indicadores de gestión. - Establecer alianzas con diferentes estamentos de acuerdo con la estrategia institucional.

3. Gestión de recursos: Gestionar la consecución de recursos con base en requerimientos del proyecto educativo. Algunas de las actividades que la persona puede realizar en esta competencia se encuentran; - Organizar proyectos de inversión según la política institucional y la normatividad vigente. - Diseñar la participación en procesos de licitación de acuerdo con la política de la institución y la normatividad vigente. - Gestionar contratos de acuerdo con la política institucional y la normatividad vigente.

4. Evaluación del Proyecto Educativo Institucional: Evaluar el proyecto educativo con base en los indicadores de gestión establecidos, las competencias asignadas y la normatividad vigente. Algunas de las actividades que la persona puede realizar en esta competencia se encuentran; - Valorar el desempeño del talento humano a su cargo de conformidad con los indicadores propuestos y la normatividad vigente. Evaluar el avance del proyecto educativo con base en indicadores Establecidos y la política institucional. - Evaluar el impacto del proyecto educativo, con base en indicadores establecidos y la política institucional.- Rendir cuentas de acuerdo con la normatividad vigente y los indicadores de gestión establecidos.- Trazar planes de mejoramiento a partir de los resultados de la evaluación institucional.

Desde lo planteado, se pueden diseñar estrategias para el abordaje puntual del aprendizaje por competencias, siendo condición el trabajar transversalmente para el logro efectivo de la formación de competencias en los estudiantes. 


\section{MÉTODO}

Se trabajó desde un tipo de investigación explicativa, definida por Hernández, Fernández, y Baptista (2014), mediante un diseño cuasi experimental de pre prueba, postprueba y grupo control, lo cual permitió determinar la efectividad de aprendizaje por competencias en ingeniería del software. La población estuvo conformada por 21 estudiantes para el grupo control y 18 para el grupo experimental de la universidad Laica Eloy Alfaro de Manabí (ULEAM), durante el período académico correspondiente al primer semestre 2019 a quienes se les aplicó el aprendizaje por competencias como estrategia didáctica en finalidad de fomentar un aprendizaje con pertinencia y significativo para la vida.

\section{RESULTADOS}

Efectividad de aprendizaje por competencias en ingeniería del software.

\section{Cuadro 1.}

\begin{tabular}{|c|c|c|c|c|c|c|}
\hline & \multicolumn{6}{|c|}{ Valor de prueba $=5$} \\
\hline & \multirow[b]{2}{*}{$t$} & \multirow[b]{2}{*}{$\mathrm{gl}$} & \multirow[b]{2}{*}{ Sig. (bilateral) } & \multirow{2}{*}{$\begin{array}{l}\text { Diferencia de } \\
\text { medias }\end{array}$} & \multicolumn{2}{|c|}{$\begin{array}{c}95 \% \text { de intervalo de confianza } \\
\text { de la diferencia }\end{array}$} \\
\hline & & & & & Inferior & Superior \\
\hline GC1 & $-11,090$ & 20 &, 000 & $-1,61905$ & $-1,9236$ & $-1,3145$ \\
\hline GE1 & $-11,985$ & 17 &, 000 & $-1,44444$ & $-1,6987$ & $-1,1902$ \\
\hline $\mathrm{GC} 2$ & $-13,645$ & 20 &, 000 & $-1,52381$ & $-1,7568$ & $-1,2909$ \\
\hline GE2 & $-1,844$ & 17 & ,083 &,- 16667 &,- 3574 &, 0240 \\
\hline
\end{tabular}

Se describe del siguiente modo:

GC1 = Grupo control en pre prueba

GE1 = Grupo experimental en pre prueba

$\mathrm{GC2}=$ Grupo control en pos prueba

GE2 = Grupo experimental en pos prueba

Se evidencia que el grupo experimental en pos prueba obtuvo 0,83 de significancia bilateral, lo cual implica que existió cambio en relación a la pre prueba, y al grupo control, por lo tanto se acepta la hipótesis verdadera: 


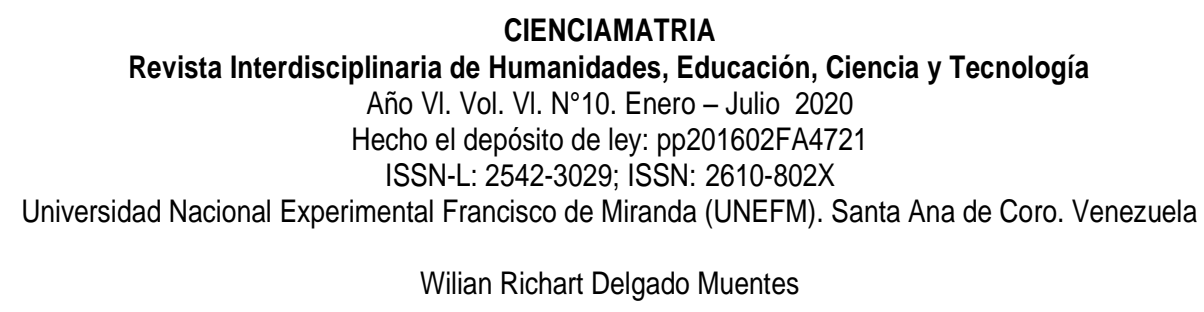

H1: Si existe efecto significativo del aprendizaje por competencias en ingeniería del software.

\section{DISCUSIÓN}

Al existir un efecto significativo se tiene que se ha generado la aplicación efectiva del aprendizaje por competencias, lo cual implica transcender el modelo tradicional de enseñanza por uno donde el estudiante se convierte en agente activo de su propio aprendizaje, teniendo relación con lo propuesto por lanni Gómez (2017), quien indica la necesidad de educar en paradigma flexible, dinámico, complejo que permita a los estudiantes formarse mediante una eficiente interrelación teoría - práctica mediante la generación de la auto reflexión como eslabón principal para asegurar el empoderamiento del aprendizaje mediante la comprensión de la realidad como un todo interconectado.

\section{CONCLUSIONES}

El aprendizaje por competencias se erige como una opción significativa para promover un aprendizaje que le permita a los estudiantes desarrollar capacidades mediante el aprender haciendo, correlacionado lo aprendido con resolución de problemas reales, así se forman con mayor pertinencia, sin embargo, esto representa un reto para la educación superior del Ecuador, tal como plantea Azuero Azuero (2019), por cuanto se debe asumir un nuevo enfoque paradigmático, siendo que la actual investigación contribuye en posicionar un nuevo modelo en favor de articular un mejor aprendizaje.

Los estudiantes objeto de estudio han desarrollado competencias genéricas como liderazgo gerencial - social en detectar y asumir el reto de solucionar una problemática real, mediante el diseño de un software para la industria atunera de Manta, mediante el cual pueden procesar estadísticamente la cantidad de desechos producidos, aprendizaje generado mediante el desarrollo de un proyecto educativo que permitió trabajar en equipo, además de implícitamente investigaron para conocer cuál era la alternativa más 


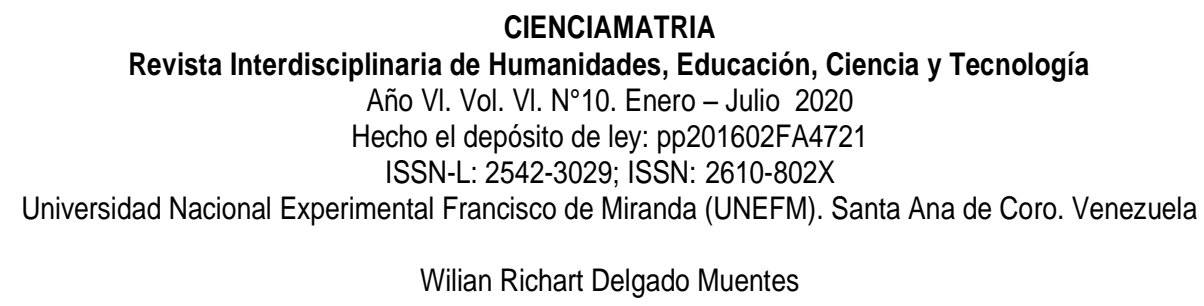

viable, adquiriendo así conocimientos, destrezas, que le permiten obtener experiencia profesional desde su formación académica.

\section{REFERENCIAS CONSULTADAS}

1. Azuero Azuero, Á. (2019). Competencias pedagógicas para el desarrollo de estrategias didácticas en estudiantes con necesidades educativas especiales en la Educación Superior Ecuatoriana. Revista Arbitrada Interdisciplinaria Koinonía, 4(7), 247-261. doi:http://dx.doi.org/10.35381/r.k.v4i7.203

2. Corominas, E. (2001). Competencias genéricas en la formación universitaria. Revista de Educación, 325, 299-321.

3. Frandeschin, T. (2018) ¿Qué es el Aprendizaje basado en competencias, y por qué se perfila como la próxima revolución educativa? Recuperado de http://edu4.me/que-es-el-aprendizaje-basado-en-competencias-y-por-que-seperfila-como-la-proxima-revolucion-educativa/

4. Ianni Gómez., L. (2017). Miramda: Una propuesta educativa emergente desde la investigación. Revista Arbitrada Interdisciplinaria Koinonía, 2(3), 9-30. Recuperado de

http://fundacionkoinonia.com.ve/ojs/index.php/revistakoinonia/article/view/49/36

5. Ministerio de Educación de El Salvador (2008). Currículo al servicio del aprendizaje. Recuperado de https://es.slideshare.net/enmaquijada/nuevo-manualde-evaluacin-de-los-aprendizajes-2014-el-salvador

6. Tobón, S. (2009). Formación basada en competencias. Pensamiento complejo, diseño curricular y didáctica. España. Universidad Complutense de Madrid.

\section{REFERENCES CONSULTED}

1. Azuero Azuero, Á. (2019). Pedagogical competences for the development of didactic strategies in students with special educational needs in Ecuadorian Higher Education. Interdisciplinary Arbitrated Review Koinonía, 4 (7), 247-261. doi: http: //dx.doi.org/10.35381/r.k.v4i7.203

2. Corominas, E. (2001). Generic competences in university education. Education Magazine, 325, 299-321. 
Wilian Richart Delgado Muentes

3. Frandeschin, T. (2018) What is competency-based Learning, and why is it emerging as the next educational revolution? Recovered from http://edu4.me/quees-el-aprendizaje-basado-en-competencias-y-por-que-se-perfila-como-laproxima-revolucion-educativa/

4. Ianni Gómez., L. (2017). Miramda: An educational proposal emerging from research. Interdisciplinary Arbitrated Review Koinonía, 2 (3), 9-30. Recovered from http://fundacionkoinonia.com.ve/ojs/index.php/revistakoinonia/article/view/49/36

5. Ministry of Education of El Salvador (2008). Curriculum at the service of learning. Recovered from https://es.slideshare.net/enmaquijada/nuevo-manual-deevaluacin-de-los-aprendizajes-2014-el-salvador

6. Tobón, S. (2009). Training based on competencies. Complex thinking, curriculum and didactic design. Spain. Complutense University of Madrid. 